\title{
Consumo de alcohol, relación con el trauma y su severidad
}

\author{
Edward Delgado*, Julio Trostchansky†, Marcelo Mion, Carlos Ketzoian§, \\ Ana Barindelli", Fernando Machado**
}

\section{Resumen}

Introducción: Uruguay no cuenta con estudios que reporten resultados de alcoholemias en traumatizados y menos aún su asociación con la severidad del trauma. Desde el año 2016 nuestro país cuenta con la llamada ley de tolerancia cero en base al supuesto de que la alcoholemia positiva representa un factor de riesgo para la severidad del trauma.

Objetivos: describir el perfil epidemiológico de los traumatizados y estudiar la asociación existente entre el consumo de alcohol y la severidad del trauma.

Material y método: estudio transversal; se incluyeron todos los pacientes que presentaron un evento traumático y fueron asistidos en el Departamento de Emergencia del Hospital de Clínicas durante el período 1 de marzo al 31 de agosto de 2017. Los datos se recopilaron prospectivamente y se extrajo muestra de alcoholemia a cada uno de los pacientes. Se consideraron diversas variables relacionadas con los pacientes y la severidad del traumatismo Se analizó la asociación entre las alcoholemias positivas y la severidad del trauma.

Resultados: se incluyeron 121 pacientes, 28\% presentaron alcoholemia positiva y $27 \%$ presentaron trauma severo. La alcoholemia positiva se asoció con la severidad del trauma $(p<0,0001)$. No se encontró asociación entre el nivel de alcoholemia y la severidad del trauma $(p=0,323)$.

Conclusiones: el consumo de alcohol se asocia significativamente con la severidad del trauma. Nuestro estudio muestra que los traumatismos son severos solo por el hecho de presentar alcoholemias positivas independientemente de su nivel, apoyando la implementación de la ley de tolerancia cero.

\begin{tabular}{|c|c|c|c|}
\hline Palabras clave: & $\begin{array}{l}\text { Consumo de bebidas alcohólicas } \\
\text { Heridas y lesiones } \\
\text { Índices de gravedad del trauma }\end{array}$ & Key words: & $\begin{array}{l}\text { Alcohol drinking } \\
\text { Wounds and injuries } \\
\text { Trauma severity indices. }\end{array}$ \\
\hline
\end{tabular}

\footnotetext{
* Cirujano general del Departamento de Emergencia, Hospital de Clínicas "Dr. Manuel Quintela".

† Prof. Adjunto del Departamento de Emergencia, Hospital de Clínicas "Dr. Manuel Quintela".

‡ Residente de Cirugía. Clínica Quirúrgica "A".

$\S$ Prof. Agregado de Métodos Cuantitativos.

II Prof. Agregada de Laboratorio Clínico.

** Profesor Titular del Departamento de Emergencia, Hospital de Clínicas "Dr. Manuel Quintela".

Facultad de Medicina. Universidad de la República

Correspondencia: Dr. Edward Delgado. Francisco Muñoz 3354 ap. 501. Montevideo. Correo electrónico: edudel.galeano@gmail.com, Tel 091218116.

Aprobado por el Comité de Ética institucional. Los autores declaran no tener conflicto de intereses.

Recibido: $11 / 3 / 19$

Aprobado: 29/4/19
} 


\section{Introducción}

El fenómeno del consumo de alcohol y otras drogas es complejo y variable. Eso implica que las soluciones puedan ser también ser complejas y variables.

Evaluar la frecuencia y el impacto del uso indebido de alcohol sobre la generación y gravedad del trauma -enfermedad traumática- es necesario para apoyar programas de prevención, disminuir su uso o hacer un uso menos riesgoso si es preciso ${ }^{(1)}$. Múltiples estudios al respecto reflejan dicha necesidad en nuestro medio y en el mundo ${ }^{(2-7)}$.

El alcohol está implicado en una amplia variedad de enfermedades, trastornos y lesiones, así como en múltiples problemas sociales y legales ${ }^{(8-10)}$.

Las lesiones por siniestros de tránsito y de peatones, las caídas, los problemas laborales a menudo se producen como consecuencia del consumo excesivo de alcohol. Los riesgos se asocian con el patrón de bebida y con la cantidad de alcohol consumido ${ }^{(11)}$. La ignorancia de los límites del consumo y de los riesgos asociados con el consumo excesivo son factores importantes. También representan un papel destacable las influencias sociales y ambientales, tales como las costumbres y actitudes que favorecen el consumo elevado.

Desde el punto de vista de la definición de políticas o intervenciones a realizar es importante, en términos de costo-efectividad, identificar con precisión y cuantificar el problema. Hemos detectado un vacío de información estadístico de la cantidad de traumatizados y la vinculación de estos con el consumo de alcohol ${ }^{(12)}$. Dicha ausencia de datos estadísticos no solo existe en el Departamento de Emergencia de nuestra institución; su ausencia se da en el resto de las instituciones asistenciales de nuestro país, públicas y privadas ${ }^{(13)}$. Salvo el reporte parcial de la Unidad Nacional de Seguridad Vial (UNASEV) referida a las espirometrías realizadas en el lugar del siniestro, no existen en nuestro país estudios y cifras nacionales fidedignas que reporten incidencia de la alcoholemia en los traumatizados y menos aún su correlación con la severidad del trauma.

Para el grupo de trabajo es de suma importancia estudiar la frecuencia del consumo de alcohol en el trauma y dentro de este específicamente en los siniestros de tránsito. Los antecedentes y datos nacionales vinculados a la siniestralidad vial desde hace muchos años, así como datos estadísticos oficiales más recientes, recabados por el Ministerio de Salud y la UNASEV en su informe $2015^{(14)}$ muestran a los siniestros de tránsito como la primera causa de muerte en menores de 35 años. Se trata de una verdadera epidemia, un verdadero problema de salud pública.

El trabajo del Dr. Rodolfo Vázquez (2004) Causas de los siniestros de tránsito desde una visión de la medi- cina social. El binomio alcohol-tránsito ${ }^{(1)}$, el cual constituye un esfuerzo interesante desde donde plantear la problemática, dejó abierto el camino para una búsqueda afinada de la medición de la frecuencia del alcohol en los siniestros de tránsito en nuestro país.

El Observatorio Uruguayo de Drogas (OUD) ha demostrado en sus últimos trabajos que la droga más consumida por los uruguayos es el alcohol, consumo que se encontraría asociado a los siniestros de tránsito ${ }^{(1)}$.

Hoy día en nuestro país existe un subregistro de los pacientes que sufren un evento traumático y que consumieron alcohol, más aún si el evento traumático fue un siniestro de tránsito.

Si analizamos los datos de UNASEV 2017, en su informe de siniestralidad vial, se presentan datos correspondientes a las espirometrías realizadas en el lugar del hecho a conductores que participaron en siniestros de tránsito. De dicho informe se desprende que menos del $7 \%$ de los conductores que participaron en siniestros de tránsito presentaron alcohol en sangre medido de forma indirecta a través de espirometría. De ellos, 0,9\% presentaba alcoholemia menor a 0,3 gramos/litro mientras que el restante 5,9\% (1.030) presentó valores superiores a dicho límite, siendo estos resultados similares a los obtenidos en el año 2015.

Si bien estos datos son de suma importancia, es evidente que existe un subregistro de pacientes, dado que no están incluidos los de mayor gravedad, pues por la entidad de las lesiones y la urgencia del traslado no fue posible hacer la espirometría a la gran mayoría de los traumatizados severos.

Por otra parte, la ausencia de una práctica profesional frecuente que incluya como un examen básico la alcoholemia a todos los pacientes traumatizados hace que tampoco exista un registro adecuado de los pacientes con patología traumática asistidos en las instituciones de salud. Lo anterior es quizá producto de la falsa creencia de que la alcoholemia requiere de una orden judicial para su realización. Esto es válido en el caso de que el juez requiera su realización con fines legales. No obstante, la alcoholemia tiene fines asistenciales y debería ser solicitada su realización por el médico tratante cuando toma contacto con el paciente, ya que el consumo de alcohol y los efectos de este sobre el organismo puede alterar las conductas diagnósticas o incluso las conductas terapéuticas que el médico considere realizar. Su resultado, en caso de no existir orden judicial para su realización, permanecerá resguardado por el secreto profesional y del paciente.

Diferentes estudios demostraron que el consumo de alcohol tiene relación con la mayor probabilidad de sufrir un trauma severo ${ }^{(15,16)}$, así como la reducción del consumo de alcohol disminuye las lesiones graves cau- 
sadas por el trauma ${ }^{(17,18)}$. Los mencionados estudios avalan la necesidad de una legislación sólida y la aplicación de medidas tendientes a disminuir el consumo de alcohol $^{(19,20)}$.

En nuestro país los niveles de alcoholemia permitidos han venido disminuyendo paulatinamente hasta llegar a la situación actual, a partir de la implementación de la ley 19.360 en la cual el nivel aceptado es de $0 \mathrm{~g} / 1$. Esta medida, junto con la creación de UNASEV y las diferentes medidas por ella implementadas, han mostrado incidencia en la disminución de la mortalidad por siniestros de tránsito en nuestro medio ${ }^{(21)}$.

\section{Objetivos}

- Describir el perfil epidemiológico de los pacientes traumatizados ingresados en el Departamento de Emergencia del Hospital de Clínicas.

- Estudiar la asociación existente entre el consumo de alcohol y la severidad del trauma.

- Estudiar la asociación existente entre el mayor consumo de alcohol y la mayor severidad en el trauma.

- Analizar la mortalidad de la serie y su relación con el alcohol.

\section{Metodología general y plan de trabajo}

\section{Población y estudio}

El estudio fue evaluado y aprobado por el Comité de Ética del Hospital de Clínicas.

Se incluyeron de forma prospectiva todos los pacientes que presentaron un evento traumático y fueron asistidos en el Departamento de Emergencia del Hospital de Clínicas durante el período 1 de marzo al 31 de agosto de 2017.

Se excluyeron otras formas de la enfermedad traumática que no tienen vinculación con la transferencia de la energía cinética en su génesis (quemados, ahogamientos) y aquellos que no consintieran participar del estudio.

Se consignaron las siguientes variables de estudio: sexo, edad, ocupación, evento traumático (tipo, topografía, severidad del trauma, alcoholemia).

Una vez ingresado el paciente en la unidad de emergencia, se realizó en formulario cerrado la recolección de las variables de estudio en forma simultánea al proceso asistencial del paciente.

Se extrajo una muestra de sangre, donde se determinó la alcoholemia como parte necesaria del proceso asistencial.

Se procedió luego a obtener el consentimiento informado para participar de la investigación. Realizados los procedimientos clínicos imagenológicos y terapéuticos correspondientes a la atención del paciente, se realizó el
Tabla 1. Distribución de frecuencia de distribución según el sexo.

\begin{tabular}{lcccc}
\hline & Frecuencia & Porcentaje & $\begin{array}{c}\text { Porcentaje } \\
\text { válido }\end{array}$ & $\begin{array}{c}\text { Porcentaje } \\
\text { acumulado }\end{array}$ \\
\hline Masculino & 99 & 81,8 & 81,8 & 81,8 \\
Femenino & 22 & 18,2 & 18,2 & 100,0 \\
Total & 121 & 100,0 & 100,0 & \\
\hline
\end{tabular}

cálculo del Injury Severity Score (ISS) en base a los hallazgos.

Definimos como traumas graves a aquellos que presentaron valores mayores a 15 en esta escala (ISS). Este índice pronóstico utilizado tiene la virtud de establecer una predicción estadística de probabilidad de supervivencia, por ende, de mortalidad, de tiempo y costos de internación y de complicaciones y secuelas evolutivas. Diversos trabajos han demostrado una relación lineal entre el porcentaje de mortalidad y los valores de ISS: para puntajes menores a 10 , la mortalidad es casi nula, mientras que 50 puntos o más equivale a una mortalidad próxima a $100 \%{ }^{(22)}$.

Durante todo el proceso asistencial se cuidaron los aspectos éticos referidos a la investigación, como ser la solicitud de consentimiento informado y la confidencialidad de los datos.

\section{Análisis estadístico}

Se realizó una descripción de las variables en estudio utilizando medidas de tendencia central y de dispersión. Para las variables cuantitativas utilizamos la media, la mediana y el desvío estándar, y para las variables cualitativas, los porcentajes.

Se estudió la normalidad de la distribución para variables cuantitativas utilizando el test de Kolgomorov-Smirnov. En función de su resultado se aplicaron tests de asociación paramétricos y no paramétricos para variables continuas. La asociación entre variables cualitativas se estudió mediante la aplicación del test de chi cuadrado o el test exacto de Fisher. El nivel de significación fue en todos los casos de $\mathrm{p}<0,05$.

\section{Resultados}

Se incluyeron 121 pacientes que reunieron los criterios de inclusión y exclusión detallados en Material y método. En la serie obtenida se observó un predomino del sexo masculino con 99 casos $(81,8 \%$ ) (tabla 1 y figura 1 ).

La enfermedad traumática afectó en nuestro estudio principalmente a la población joven, con una media de edad de 35 años. 


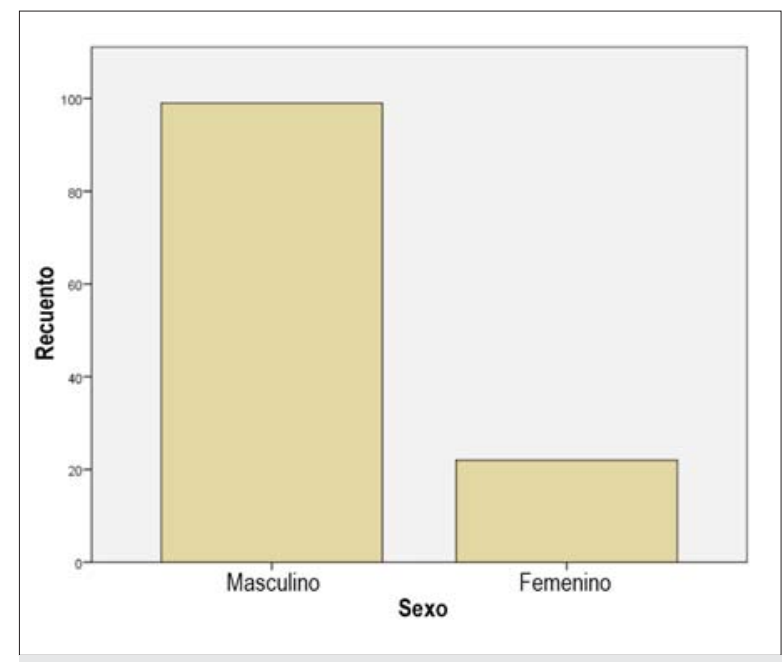

Figura 1. Sexo. Valores absolutos.

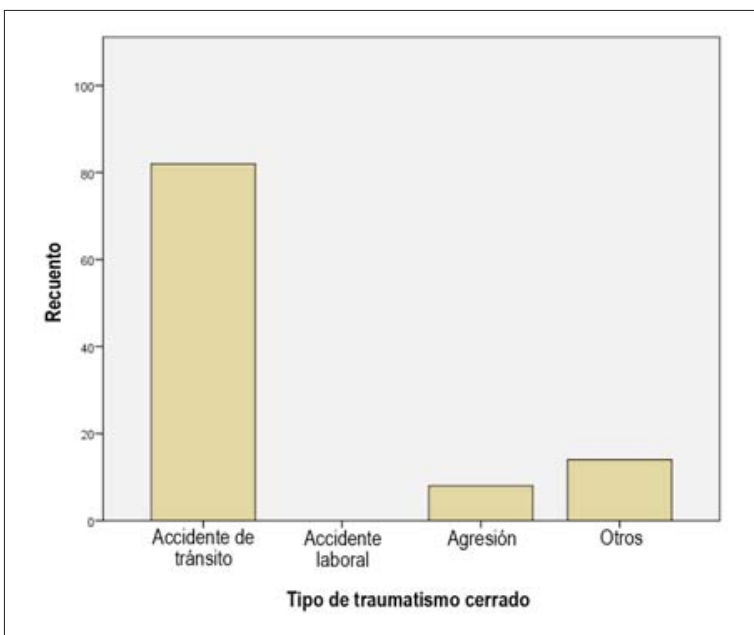

Figura 2. Tipos de traumatismo cerrado. Valores absolutos.

En relación con el tipo de trauma la mayoría correspondió a traumas cerrados ( $86 \%$ ), y dentro de estos al siniestro de tránsito ( $82 \%$ de los traumas cerrados) (tablas 2 y 3 , figura 2 ).

Si analizamos los traumas penetrantes, que constituyeron el 14\% del total, la mayoría correspondió a heri-
Tabla 2. Tipos de traumatismo. Del total de la serie el $86 \%$ correspondieron a traumas cerrados 104 casos, los restantes 17 fueron traumas penetrantes.

\begin{tabular}{lcrrc}
\hline & Frecuencia & Porcentaje & $\begin{array}{c}\text { Porcentaje } \\
\text { válido }\end{array}$ & $\begin{array}{c}\text { Porcentaje } \\
\text { acumulado }\end{array}$ \\
\hline No & 17 & 14,0 & 14,0 & 14,0 \\
Si & 104 & 86,0 & 86,0 & 100,0 \\
Total & 121 & 100,0 & 100,0 & \\
\hline
\end{tabular}

Tabla 3. Causas determinantes de traumatismos cerrados

\begin{tabular}{lcccc}
\hline & Frecuencia & $\begin{array}{c}\text { Porcentaje } \\
\text { (\%) }\end{array}$ & $\begin{array}{r}\text { Porcentaje } \\
\text { válido (\%) }\end{array}$ & $\begin{array}{c}\text { Porcentaje } \\
\text { acumulado } \\
\text { (\%) }\end{array}$ \\
\hline Siniestro de tránsito & 82 & 67,8 & 78,8 & 78,8 \\
Agresión & 8 & 6,6 & 7,7 & 86,5 \\
Otros & 14 & 11,6 & 13,5 & 100,0 \\
Total & 104 & 86,0 & 100,0 & \\
\hline
\end{tabular}

das de arma de fuego (53\%), seguidas de heridas de arma blanca (47\%) (tabla 4, figura 3).

El valor de la alcoholemia se obtuvo en la totalidad de los casos, siendo la mayoría alcoholemias negativas (72\%); las alcoholemias positivas correspondieron al $28 \%$ de la serie (34 casos) (figura 4 ).

De acuerdo con el ISS, la cantidad de traumas severos correspondió a $27 \%$ (figura 5).

Se analizó la asociación entre las alcoholemias positivas y la severidad del trauma obteniéndose una asociación estadísticamente significativa (mayor severidad de trauma en pacientes con alcoholemia positiva, test de $\mathrm{U}$ de Mann Whitney, $\mathrm{p}<0,0001$ ) (prueba paramétrica, anexo 1).

No se encontró correlación entre el nivel de alcoholemia y la severidad del trauma medida por la escala ISS

Tabla 4. Causas determinantes de traumas penetrantes.

\begin{tabular}{llcccc}
\hline & & Frecuencia & Porcentaje & Porcentaje válido & Porcentaje acumulado \\
\hline Válido & Arma blanca & 8 & 6,6 & 47,1 & 47,1 \\
& Arma de fuego & 9 & 7,4 & 52,9 & 100,0 \\
& Total & 17 & 14,0 & 100,0 & \\
\hline
\end{tabular}




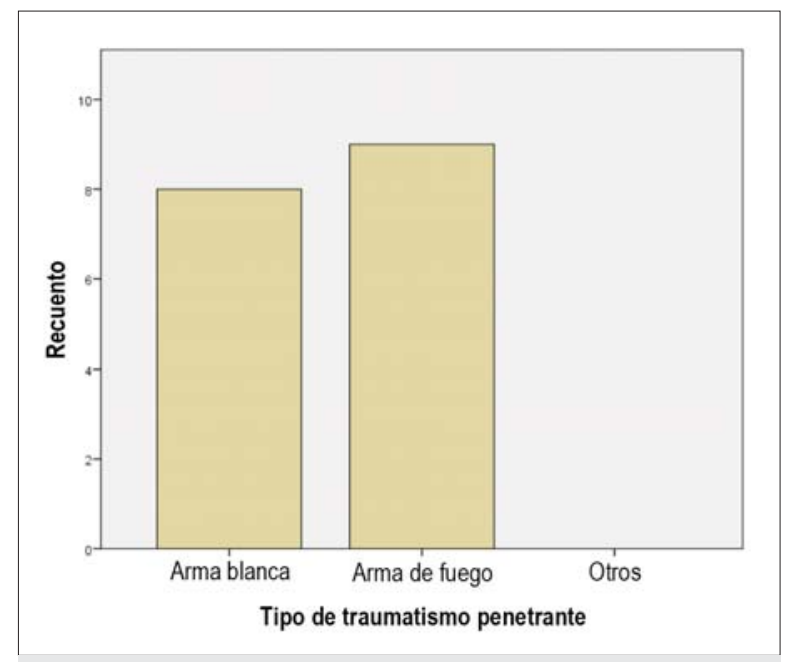

Figura 3. Tipos de traumatismo penetrante. Valores absolutos.

(test de correlación no paramétrico de Spearman, $\mathrm{p}=$ 0,323) (prueba no paramétrica, anexo 2).

\section{Discusión}

En el presente estudio se analizaron los efectos del alcohol en relación con el trauma para los usuarios que consultaron en el Departamento de Emergencia del Hospital de Clínicas durante el período comprendido entre el 1 de marzo al 31 de agosto de 2017.

Hasta la fecha, en nuestro país, este es el estudio transversal más grande enfocado en el tema en la literatura.

No contamos con datos de registros de alcoholemias en las puertas de emergencia de los diferentes centros asistenciales, mucho menos de datos que afirmen si existe asociación entre el consumo de alcohol y la posibilidad de sufrir un trauma severo.

El estudio presentado surge de la necesidad de describir las características epidemiológicas de los traumatizados, estudiar y analizar la asociación existente entre el consumo de alcohol y la severidad del trauma, y analizar la asociación entre el mayor consumo de alcohol y la mayor severidad en el trauma, para poder así implementar, entre otros puntos, medidas de prevención tendientes a evitar el consumo de alcohol.

Como sabemos, el alcohol es uno de los factores de riesgo personales más importantes para sufrir una lesión grave o mortal. Esto contribuye aproximadamente a un tercio de todas las muertes por siniestros y por trauma en general $^{(23)}$.

Más del 90\% de estas muertes se dan en países subdesarrollados o en vías de desarrollo, el gasto producido por esta pandemia, la enfermedad trauma, corresponde a entre $1 \%$ y $3 \%$ del PBI.

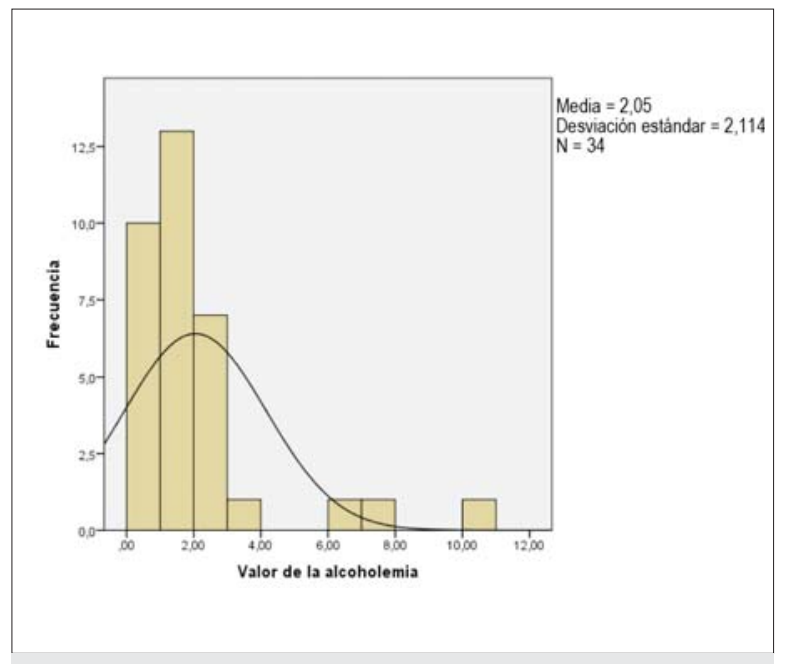

Figura 4. Alcoholemias. 34 casos positivos. $28 \%$ de la serie.

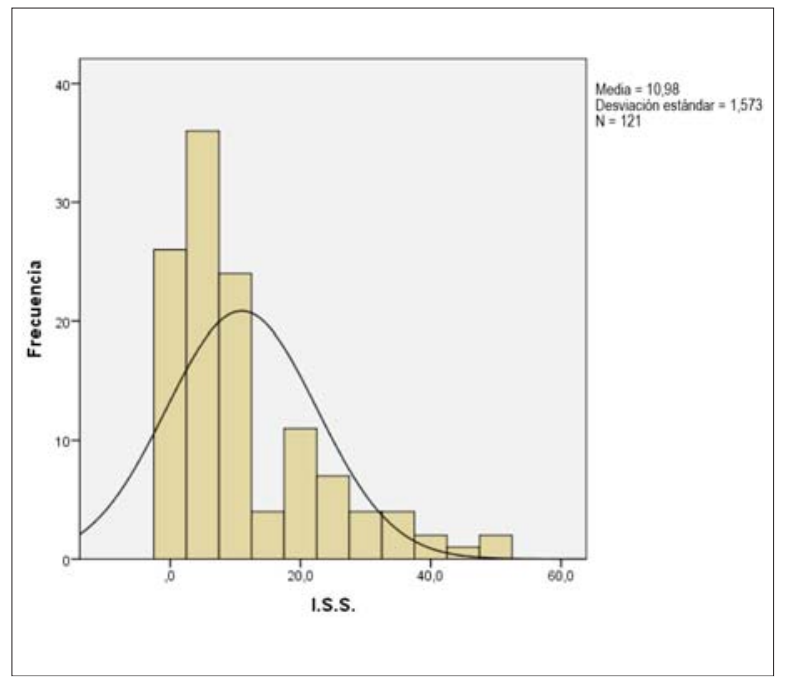

Figura 5. Severidad del trauma. 33 casos fueron traumas severos $(27,2 \%)$.

La realidad de Uruguay ha ido cambiando tanto en la mortalidad por trauma y dentro de este específicamente en los siniestros de tránsito, siendo imperantes las acciones destinadas a controlar los factores de riesgo.

Sabemos, por datos de la UNASEV, que menos de $6,7 \%$ de las espirometrías fueron positivas en el lugar del siniestro (UNASEV, 2017). Datos internacionales refieren que el alcohol está presente en más de la mitad de los siniestros mortales ${ }^{(24,25)}$.

Destacamos el subregistro de las espirometrías, dado que las mismas son realizadas en el lugar del hecho, no contamos con datos de siniestros graves o fatales, ya que estos pacientes son trasladados rápidamente a las puertas de emergencia. 
En nuestro estudio detectamos $28 \%$ de alcoholemias positivas que correspondieron a 34 casos, esta mayor incidencia respecto de la bibliografía ${ }^{(13,15)}$ se debió probablemente a que analizamos una población que requirió un rápido traslado por su tipo de trauma a un centro hospitalario. Como fue demostrado en este trabajo, los traumatizados con traumas más severos tienen mayor relación con el consumo de alcohol, por lo que era esperable que las cifras fueran superiores.

Para confirmar y cuantificar la intoxicación por alcohol utilizamos la medición de alcoholemias. Algunos estudios demostraron que el compromiso neurológico como predictor de intoxicación alcohólica se correlacionó pobremente con los niveles de alcoholemia en sangre $^{(26)}$. La evaluación clínica como único elemento no identifica correctamente la intoxicación aguda o crónica de alcohol ${ }^{(27)}$. El 31\% de los pacientes gravemente heridos están intoxicados, así como 33\% de los pacientes ventilados, y $37 \%$ de los pacientes en estado de shock, un grupo en el cual es difícil valorar clínicamente la intoxicación $^{(28)}$. Diversos estudios han mostrado que solo en centros de trauma consistentemente se realizan alcoholemias a los traumatizados ${ }^{(29)}$. La medición de alcoholemia en sangre debe ser propuesta para todos los pacientes con lesiones agudas porque las intervenciones agudas pueden tener un impacto en el resultado asistencial $^{(28)}$.

Respecto de las características epidemiológicas de los pacientes, analizamos 121 casos, observando un predominio del sexo masculino (99 casos, 81,8\%) (tabla 1), siendo esto acorde a lo encontrado en la mayoría de los estudios ${ }^{(13,15,23)}$.

La enfermedad traumática afectó en nuestro estudio principalmente a la población joven (media para la edad 35 años, en concordancia con lo que sucede en el resto de los países) ${ }^{(30,31) \text {. }}$

En relación con el tipo de trauma la mayoría correspondió a traumas cerrados $(86 \%)$, y dentro de estos al siniestro de tránsito $(82 \%)$ (tabla 2).

$\mathrm{Si}$ analizamos los traumas penetrantes que constituyeron el 14\% del total, los vinculados a heridas de arma de fuego constituyeron el $53 \%$, seguido de heridas de arma blanca 47\% (tabla 3), notando una leve tendencia a favor del trauma penetrante por arma de fuego.

Nuestra hipótesis planteaba que la intoxicación por alcohol conduce a un ISS (puntaje de severidad de la lesión) más alto en comparación con pacientes con alcoholemias negativas, como fue demostrado ya en otros estudios $^{(17,18)}$. Para esto comparamos los pacientes con alcoholemias positivas y negativas respecto de los índices de severidad del trauma y obtuvimos una asociación estadísticamente significativa (mayor severidad de trauma en pacientes con alcoholemia positiva, test de $U$ de
Mann Whitney, $\mathrm{p}<0,0001)$ (prueba paramétrica). Este resultado es de capital importancia en nuestra investigación, ya que refleja la problemática del consumo de alcohol en nuestro medio, así como un insumo muy importante que refuerza las medidas adoptadas de tolerancia cero.

Otros estudios han mostrado resultados diferentes al nuestro y no encontraron diferencias en la gravedad de las lesiones medidas por ISS para pacientes con alcoholemias positivas ${ }^{(32,33)}$. La mortalidad en pacientes con alto grado de lesión encefálica traumática y alcoholemia positiva fue menor en otras series ${ }^{(32)}$.

Andersen y colaboradores examinaron la correlación entre los niveles de alcohol y la tasa de conductores de automóviles con y sin cinturón de seguridad. Encontraron que el 51\% de los pacientes sin cinturón y el 21\% de los pacientes con cinturón tenían una alcoholemia positiva; concluyeron que la mayor gravedad de la lesión en pacientes alcoholizados puede relacionarse con la conducción de automóviles sin cinturón de seguridad, los conductores subestiman el riesgo producto de la ingesta de alcohol ${ }^{(34)}$. Todos estos estudios presentaron en común revisiones de bases retrospectivas, con espirometrías realizadas en el lugar del siniestro y la no realización de alcoholemias en el centro hospitalario, como en nuestra serie, analizaron el ISS según datos recabados de historias clínicas, siendo todo ello motivo de sesgos que pensamos que pudieron influir en las conclusiones.

$\mathrm{Si}$ analizamos los subgrupos en nuestro estudio, no se encontró correlación entre el nivel de alcoholemia y la severidad del trauma medida por la escala ISS (test de correlación no paramétrico de Spearman, $\mathrm{p}=0,323$ ) (prueba no paramétrica) si bien existen artículos, con diferentes problemas de diseño, que sí lo demues$\operatorname{tran}^{(17,31,33)}$

Nuestros resultados dejan claro que los traumas severos no son más severos a mayor consumo de alcohol, son severos solo por el hecho de presentar una alcoholemia positiva, aunque su medida sea baja. Existieron traumas severos con bajos niveles de alcoholemia y traumas leves con niveles altos de alcoholemia. Dicho de otra manera: la posibilidad de un trauma severo estaba dada por el solo hecho de haber tomado alcohol independientemente de su cantidad. Esto refuerza lo establecido en la ley 19.360, de tolerancia cero para el consumo de alcohol.

Muchas de las lesiones y muertes ocurren mientras los conductores están intoxicados o bajo la influencia del alcohol. Numerosos estudios como el nuestro han tenido como objetivo evaluar la relación del alcohol con el trauma y la instalación de programas continuos de intervención para reducir la incidencia de lesiones. 
La evidencia disponible sugiere que los profesionales, los educadores y la comunidad en su conjunto deben continuar el esfuerzo en las estrategias de prevención del consumo de alcohol. En nuestro hospital se implementó un nuevo programa donde a todos los pacientes tratados en nuestro centro con alcoholemia positiva durante la hospitalización se les realizó una prueba breve de alcohol y, en función del puntaje de riesgo, se implementó la intervención derivando a desintoxicación como paciente internado o recibiendo una cantidad de recursos para luego del alta. El resultado de dicha intervención será motivo de una comunicación futura.

Los centros de trauma pueden y deben aumentar estos esfuerzos mediante la participación constante en exámenes de detección, intervención breve y derivación para el tratamiento. Creemos que esto impactará de forma satisfactoria en la prevención de futuros eventos traumáticos. Aumentar los esfuerzos al tomar contacto con estos pacientes en el departamento de emergencia y detectar el consumo de alcohol salvará vidas y también reducirá los costos generales de salud.

Algunas de las limitaciones de nuestro estudio son el hecho de no ser un ensayo clínico controlado y aleatorizado, y, por lo tanto, los resultados pueden ser exagerados por factores de confusión y sesgos. De todas formas consideramos que un estudio de ese tipo puede ser de difícil implementación, ya que éticamente entendemos que corresponde realizar alcoholemia a todos los pacientes traumatizados con el fin de lograr un mejor proceso asistencial debido a las implicancias clínicas mencionadas previamente. Por otra parte, hay que tener en cuenta que otras drogas, además del alcohol (es decir, cocaína, benzodiazepinas, marihuana), podrían ser consumidas por los pacientes analizados en nuestro informe. El Centro de Control de Enfermedades de Estados Unidos anunció que la prevalencia de la intoxicación por drogas era igual en porcentaje que para la intoxicación por alcohol (Centros para Enfermedades y Prevención, 2006). Es posible que otras drogas, que no sea el alcohol, hayan tenido un efecto en el mecanismo de la lesión traumática; sin embargo, estas no fueron medidas en nuestro trabajo.

\section{Conclusiones}

Nuestro estudio permitió describir en el período analizado el perfil epidemiológico de los pacientes traumatizados ingresados en la emergencia del Hospital de Clínicas.

Encontramos que el consumo de alcohol representa por sí mismo un factor que se asocia significativamente con la severidad del trauma independientemente de los niveles de alcoholemia.
De estos datos concluimos que la prevención debe orientarse a la ausencia de consumo de alcohol en los traumatizados en consonancia con la ley de tolerancia cero implementada en nuestro país, dado que la severidad del trauma no depende de la cantidad de alcohol consumido.

\section{Abstract}

Introduction: our country has not conducted studies to report results on blood alcohol levels in traumatized patients or on its association with the severity of the traumatic injury.

Objective: to describe the epidemiological profile of trauma patients and to study the relationship between alcohol consumption and the severity of the traumatic injury.

Method: transversal study including all patients who suffered a traumatic event and were assisted at the Emergency Department of the Clínicas Hospital between March 1, 2017 and August 31, 2017. Data was prospectively collected and a blood alcohol level sample was taken from each one of the patients. Several patient related variables were considered, as well as the severity of the traumatic injury.

Results: 121 patients were included in the study, $28 \%$ showed positive blood alcohol levels and $27 \%$ presented severe traumatic injuries. Positive blood alcohol levels were associated to severity of the traumatic injury $(p<0.0001)$. No association was found between blood alcohol levels and the severity of the traumatic injury (p $=0.323$ )

Conclusions: alcohol consumption is significantly associated to the severity of trauma. Our study shows that traumas are severe simply because blood alcohol levels are positive, regardless of the alcohol level, what supports the Zero Tolerance Law.

\section{Resumo}

Introdução: no Uruguai não existem estudos que mostrem não somente os resultados de alcoolemia em traumatizados como também sua associação com a gravidade do trauma. Desde 2016 conta com a Lei de Tolerância 0 baseada no princípio de que a alcoolemia positiva é um fator de risco para a gravidade do trauma.

Objetivos: descrever o perfil epidemiológico dos traumatizados e estudar a associação existente entre o consumo de álcool e a gravidade do trauma.

Materiais e métodos: estudo transversal que incluiu todos os pacientes que apresentaram um evento traumático e foram atendidos no Departamento de Emergência do Hospital de Clínicas no período 1 de março - 31 de agosto de 2017. Os dados foram levantados prospectivamente e uma amostra de alcoolemia foi coletada a todos os pacientes. Foram incluídas variáveis relacionadas aos 
pacientes e à gravidade do traumatismo. A associação entre as alcoolemias positivas e a gravidade do trauma foi analisada.

Resultados: foram incluídos 121 pacientes; $28 \%$ apresentaram alcoolemia positiva e $27 \%$ apresentaram trauma severo. As alcoolemias positivas estavam associadas com a gravidade do trauma $(\mathrm{p}<0,0001)$. Os resultados não mostraram associação entre o nível de alcoolemia e a gravidade do trauma $(\mathrm{p}=0,323)$.

Conclusões: o consumo de álcool está significativamente associado com a gravidade do trauma. Nosso estudo mostra que os traumatismos são graves somente pela presença de alcoolemias positivas independentemente de seu nível o que respalda a implementação da Lei de Tolerância 0.

\section{Bibliografía}

1. Vázquez Pedrouzo RA. Causas de los accidentes de tránsito o desde una visión de la medicina social: el binomio alcohol-tránsito. Rev Méd Urug 2004; 20(3):178-86.

2. Uruguay. Junta Nacional de Drogas. Incidencia del alcohol en los siniestros de tránsito en el Uruguay: generación de un Sistema de Información Permanente. Informe final julio de 2007. Montevideo: OSMET-OUD, 2007.

3. Gómez Restrepo C, Gómez García M, Naranjo S, Rondón M, Acosta Hernández A. Alcohol consumption as an incremental factor in health care costs for traffic accident victims: evidence in a medium sized Colombian city. Accid Anal Prev 2014; 73:269-73. DOI: 10.1016/j.aap.2014.09.023.

4. Campos V, de Souza e Silva R, Duailibi S, dos Santos J, Laranjeira $\mathbf{R}$, et al. The effect of the new traffic law on drinking and driving in São Paulo, Brazil. Accid Anal Prev 2013; 50:622-7. DOI: 10.1016/j.aap.2012.06.011.

5. Hall W, Wallace A, Cobiac L, Doran C, Vos T. How can we reduce alcohol-related road crash deaths among young Australians? Med J Aust 2010; 192(8):464-6.

6. de Pena M. Accidentes de trainsito y alcohol: aspectos legales y eiticos. Rev Méd Urug 1995; 11 (3): 153-6.

7. Dapueto J, Yusim S, Russi D. Utilizacioìn de cuestionarios en el diagnoistico de alcoholismo en un hospital general. Rev Méd Urug 1992; 8(2):120-6.

8. Anderson P, Cremona A, Paton A, Turner C, Wallace P. The risk of alcohol. Addiction 1993; 88(11):1493-508.

9. Edwards G, Anderson P, Babor T, Casswell S, Ferrence R, Giesbrecht N, et al. Alcohol policy and the public Good. Oxford, UK: Oxford University Press, 1995.

10. World Health Organization. WHO Expert Committee on Problems Related to Alcohol Consumption: second report. (WHO Technical Report Series; $n^{\circ}$ 944). Geneva: WHO, 2007.

11. Babor T, Higgins Biddle J, Saunders J, Monteiro M. AUDIT: cuestionario de identificación de los trastornos debidos al consume de alcohol. Pautas para su utilización en atención primaria. Ginebra: OMS, 2001. Disponible en:
www.who.int/substance_abuse/activities/en/AUDITmanualSpanish.pdf. [Consulta: 2019].

12. Barrios Camponovo G. Enfermedad traumaitica en Uruguay: aspectos epidemioloìgicos. Rev Méd Urug 1995; $11(3): 187-207$.

13. Trostchansky J, Sánchez G, Dibarboure P, Bado J, Machado F, Castiñeiras $\mathbf{S}$, et al. Historia clínica para trauma. Registro hospitalario específico para pacientes traumatizados: un recurso para países en desarrollo. Rev Méd Urug 2011; 27(1):12-20.

14. Uruguay. Unidad de Seguridad Vial. Siniestralidad vial en Uruguay: informe anual 2014. Montevideo: UNASEV, 2014. Disponible en: https://www.gub.uy/unidad-nacional-seguridad-vial/datos-y-estadisticas/estadisticas/2014-informe-anual-de-siniestralidad-vial. [Consulta: 2019].

15. Stübig T, Petri M, Zeckey C, Brand S, Müller C, Otte D, et al. Alcohol intoxication in road traffic accidents leads to higher impact speed difference, higher ISS and MAIS, and higher preclinical mortality. Alcohol 2012; 46(7):681-6. DOI: 10.1016/j.alcohol.2012.07.002.

16. Hsieh C, Su L, Wang Y, Fu C, Lo H, Lin C. Does alcohol intoxication protect patients from severe injury and reduce hospital mortality? The association of alcohol consumption with the severity of injury and survival in trauma patients. Am Surg 2013; 79(12):1289-94.

17. Fell J, Voas R. The effectiveness of a 0.05 blood alcohol concentration (BAC) limit for driving in the United States. Addiction 2014; 109(6):869-74. DOI: 10.1111/add.12365.

18. Ditsuwan V, Lennert Veerman J, Bertram M, Vos T. Cost-effectiveness of interventions for reducing road traffic injuries related to driving under the influence of alcohol. Value Health 2013; 16(1):23-30. DOI: 10.1016/j.jval.2012.08.2209.

19. Sousa T, Lunnen J, Gonçalves V, Schmitz A, Pasa G, Bastos T, et al. Challenges associated with drunk driving measurement: combining police and self-reported data to estimate an accurate prevalence in Brazil. Injury 2013; 44(Suppl 4):S11-6.

20. Carter P, Flannagan C, Bingham C, Cunningham R, Rupp J. Modeling the injury prevention impact of mandatory alcohol ignition interlock installation in all new US vehicles. Am J Public Health 2015; 105(5):1028-35.

21. Vanerio P, Trostchansky J, Machado F, Barrios G. Impacto de la ley uruguaya en la mortalidad por siniestros de tránsito. Rev Méd Urug 2018; 34(3):149-54.

22. Barrios G. Índices de gravedad em el paciente traumatizado. En: Juambeltz C, Machado F, Trostchansky J, eds. Trauma la enfermedad del nuevo milenio. Montevideo: Arena, 2005:98-9.

23. Sánchez G, Valsangiácomo $\mathbf{P}$, Trostchansky J, Machado F. Perfil epidemiológico de traumatizados graves en un hospital de agudos. Rev Méd Urug 2006; 22(3):179-84.

24. Toledo Castillo F. Manual de seguridad vial: factor alcohol. Pamplona, Navarra: Aranzadi, 2009. 
25. Dornewald H. Estudio estadístico sobre muertes en accidentes de tránsito en CABA y su relación con la presencia de alcohol en sangre cadavérica. Prensa Méd Argent 2008; 95(10):606-13.

26. Liu S, Siegel P, Brewer R, Mokdad A, Sleet D, Serdula M. Prevalence of alcohol-impaired driving. Results from a national self-reported survey of health behaviors. JAMA 1997; 277(2):122-5.

27. Danielsson P, Rivara F, Gentilello L, Maier R. Reasons why trauma surgeons fail to screen for alcohol problems. Arch Surg 1999; 134(5):564-8.

28. Maier RV. Ethanol abuse and the trauma patient. Surg Infect (Larchmt) 2001; 2(2):133-41.

29. Fabbri A, Marchesini G, Morselli-Labate A, Rossi F, Cicognani A, Dente M, et al. Positive blood alcohol concentration and road accidents. A prospective study in an Italian emergency department. Emerg Med J 2002; 19(3):210-4.
30. Abdel-Aty M, Abdelwahab H. Exploring the relationship between alcohol and the driver characteristics in motor vehicle accidents. Accid Anal Prev 2000; 32(4):473-82.

31. Sorrentino C, Schulz J, Chaudry A, Cohen E, Friedman Y. The legal limit: differences in injury severity score for vehicular crashes. J Trauma Nurs 2016; 23(4):184-8.

32. Friedman LS. Complications associated with blood alcohol concentration following injury. Alcohol 2014; 48(4):391-400. DOI: 10.1016/j.alcohol.2014.01.008.

33. Leonard E, Buckley T, Curtis K. Impact of alcohol on outcomes in hospitalized major trauma patients: a literature review. J Trauma Nurs 2016; 23(2):103-14.

34. Andersen J, McLellan B, Pagliarello G, Nelson W. The relative influence of alcohol and seatbelt usage on severity of injury from motor vehicle crashes. J Trauma 1990; 30(4):415-7. 
Anexo 1. Pruebas no paramétricas:

Análisis del consumo de alcohol (alcoholemias positivas) respecto de la severidad del trauma.

\section{Resumen de contrastes de hipótesis}

\begin{tabular}{|lll|}
\hline \multicolumn{1}{|c}{ Hipótesis nula } & \multicolumn{1}{c|}{ Prueba } & Sig. \\
\hline $\begin{array}{l}\text { La distribución de ISS es la } \\
\text { misma entre las categorias } \\
\text { de alcoholemia }\end{array}$ & $\begin{array}{l}\text { Prueba Ude } \\
\text { Mann-Whitney } \\
\text { para muestras } \\
\text { independientes }\end{array}$ & 0,000 \\
\hline
\end{tabular}

Se muestran significaciones asintóticas. El nivel de significancia es 0,0

Cuando la alcoholemia es positiva la probabilidad de sufrir un trauma severo aumenta respecto de las alcoholemias negativas. P 0,001

Anexo 2. Correlaciones no paramétricas

\begin{tabular}{|c|c|c|c|c|}
\hline & & & Valor de la alcoholemia & ISS \\
\hline \multirow[t]{6}{*}{ Rho de Spearman } & Valor de la alcoholemia & Coeficiente de correlación & 1,000 & 0,175 \\
\hline & & Sig. (bilateral) & . & 0,323 \\
\hline & & $\mathrm{N}$ & 34 & 34 \\
\hline & I.S.S. & Coeficiente de correlación & 0,175 & 1,000 \\
\hline & & Sig. (bilateral) & 0,323 & . \\
\hline & & $\mathrm{N}$ & 34 & 121 \\
\hline
\end{tabular}

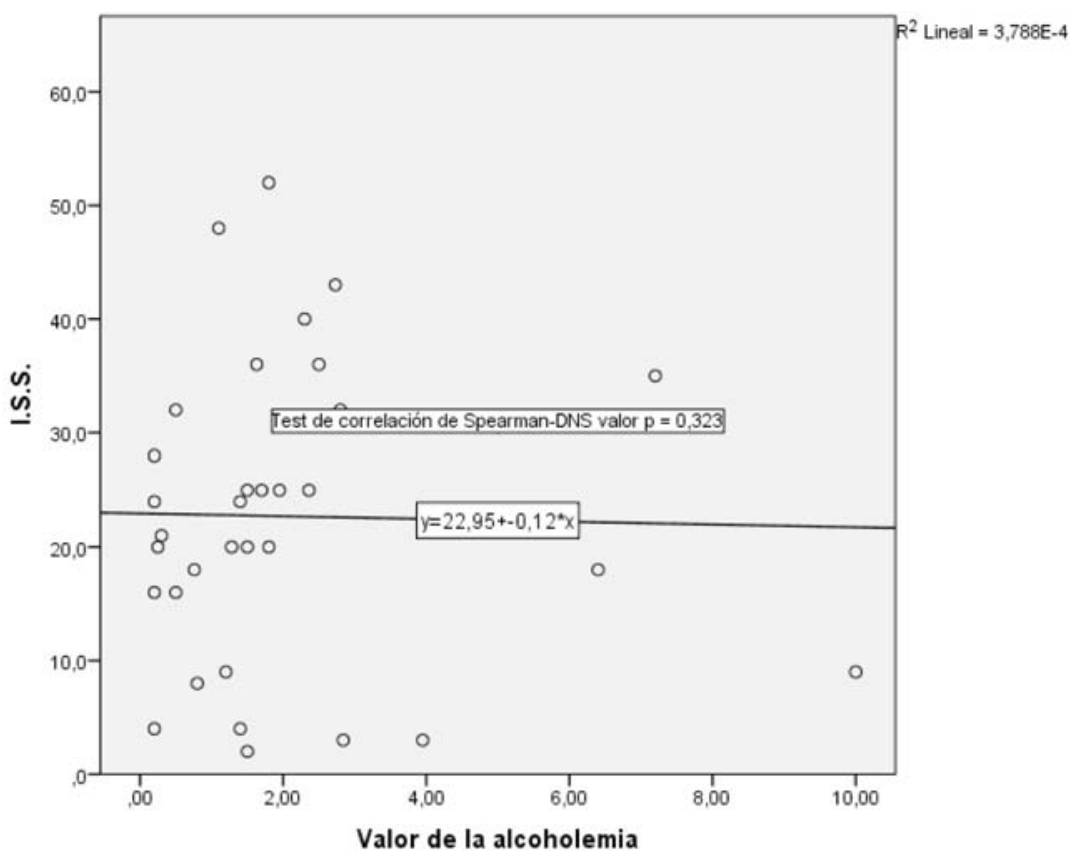

